Radial and Nonradial Pulsations as Probes of Stellar Physics

ASP Conference Series, Vol. 259, 2002

C. Aerts, T.R. Bedding, \& J. Christensen-Dalsgaard, eds.

\title{
Applications of Non-Adiabatic Radial Pulsation Equations to roAp Stars
}

\author{
R. Medupe
}

South African Astronomical Observatory, PO Box 9, Observatory 7935, South Africa

Department of Physics, University of North West, Mmabatho 2735, South Africa

Department of Astronomy, University of Cape Town, Rondebosch 7701, South Africa

\section{J. Christensen-Dalsgaard}

Teoretisk Astrofysik Center, Aarhus Universitet, DK-8000 Aarhus C, Denmark

D.W. Kurtz

Centre for Astrophysics, University of Central Lancashire, Preston PR1 2HE, UK

\begin{abstract}
We apply a code that solves grey radial non-adiabatic pulsation equations with consistent treatment of radiative transfer to the roAp frequency regime. We find evidence for the existence of chromospheric modes at frequencies $2.5 \mathrm{mHz}, 3.7 \mathrm{mHz}, 4.6 \mathrm{mHz}$ and $5.7 \mathrm{mHz}$ in the equilibrium model we used. We also find that, since the oscillations are not adiabatic at the surfaces of roAp stars, better surface boundary conditions need to be considered in future studies.
\end{abstract}

\section{Introduction}

In this paper, we consider radial oscillations in the atmospheres of roAp stars in the grey case. Since roAp stars are high overtone pulsators, their pulsations occur quite close to their surfaces and are therefore non-adiabatic. The nonadiabaticity comes as a result of energy being radiated away into space at the stellar surface. Our treatment of energy includes radiative transfer as discussed in Christensen-Dalsgaard \& Frandsen (1983); convective and magnetic effects as well as rotation are not included. The high pulsation frequencies of the roAp stars imply that our results for radial oscillations can also be taken as representative for non-radial oscillations, in the near-surface region where nonadiabatic effects are important.

The roAp stars are introduced by Cunha and Weiss (these proceedings). Balmforth et al. (2001) showed that magnetic fields at the magnetic poles can suppress convection sufficiently to allow high-overtone pulsations to be excited 
by the $\kappa$ mechanism operating in the hydrogen ionization zone. Therefore, interpretations of the results presented here should bear Balmforth et al.'s results in mind as well.

Our study was motivated by an attempt to understand the large variation of pulsation phase with wavelength observed in HD 101065 and HR 3831 (Medupe et al., 2001) since Medupe et al. (1998) show that current analytical pulsation models, such as the one presented by Watson (1988), fail to explain the roAp multi-colour data.

\section{Non-adiabatic radial oscillation calculations}

Our treatment of the oscillation equations is similar to that of ChristensenDalsgaard \& Frandsen (1983). The atmosphere is treated as plane parallel and is matched to a spherically symmetric envelope. The oscillation equations include the perturbed continuity and momentum equations in the atmosphere. There are also two radiation equations derived from the equation of radiative transfer. The equations in the atmosphere form an unclosed system containing four first-order differential equations with five variables, namely, the displacement, $\delta r$, relative pressure perturbations, $\delta P / P$, Eddington flux perturbations, $\delta H$, the mean intensity perturbations, $\delta J$, and perturbations in the second moment of the transfer equation, $\delta K$. The equations are closed by introducing the variable Eddington factors $f_{o s c}(r)=\delta K(r) / \delta J(r)$ and $g_{o s c}=\delta H\left(r_{s}\right) / \delta J\left(r_{s}\right)$; here $r_{s}$ is the surface radius of the model. In the envelope, the equations, including the diffusion approximation and the energy equation in addition to the continuity and momentum equations, form a closed fourth-order system. We imposed three boundary conditions at the surface, four matching conditions and a single boundary condition at the base of the envelope. The full set is iterated with initial values of $f_{o s c}$ and $g_{o s c}$ taken as the equilibrium values of the Eddington factors.

We checked our code successfully against the asymptotic theory and verified that the oscillations become adiabatic below the photosphere and throughout the envelope.

\section{The damping rates}

The calculations presented here are based on an equilibrium model with surface temperature $T_{\text {eff }}=9237.5 \mathrm{~K}$ and gravity $\log g=4.33$. It has solar abundances and is assumed to be chemically homogeneous.

The damping rates were calculated by requiring that the model beneath the base of the envelope performs no work on the part above it. To implement this we selected the real part of the cyclic frequency $(\omega)$ and kept it constant, regarding the imaginary part as the eigenvalue. The result of this at several frequencies is shown in Fig. 1. It is clear that all the radial modes in the roAp frequency regime $(1 \mathrm{mHz}<\nu<3 \mathrm{mHz})$ are damped. The results are similar to those found by Balmforth et al. (2001) in their equatorial model. We also see an increased damping near the acoustic cut-off frequency. This is expected since modes leak out and are damped here. When all modes are reflected back into the star, this peak is not seen. We then calculated the ratio of surface to total 


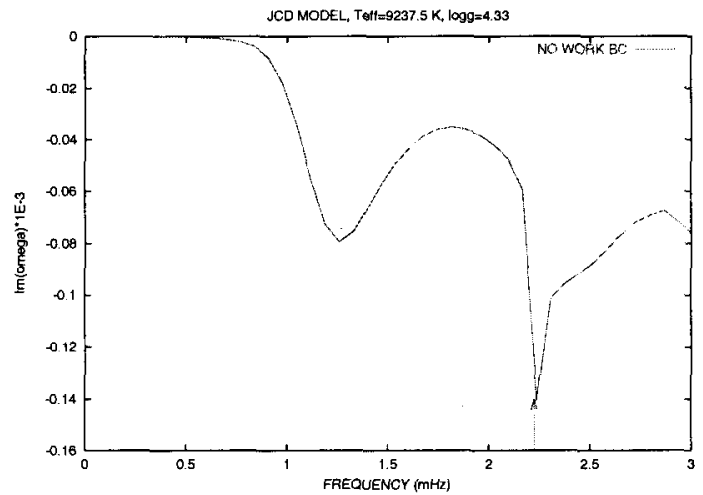

Figure 1. The boundary condition used here allows the modes with frequencies larger than the acoustic cut-off frequencies to leak out and be damped. The arrow indicates the acoustic cut-off frequency for the equilibrium model we used.

energy losses, $\eta_{s} / \eta$, in the star. The ratio $\left|\eta_{s} / \eta\right|$ is shown in the top panel of Fig. 2 for the case in which all the modes are reflected back into the star and are not allowed to leak though the surface. The bottom panel shows the normalised pulsation energy. We can see peaks in $\left|\eta_{s} / \eta\right|$ at frequencies $2.5 \mathrm{mHz}, 3.7 \mathrm{mHz}$, $4.6 \mathrm{mHz}$ and $5.7 \mathrm{mHz}$. Each of these peaks corresponds to a minimum in the pulsation energy. This indicates a chromospheric mode.
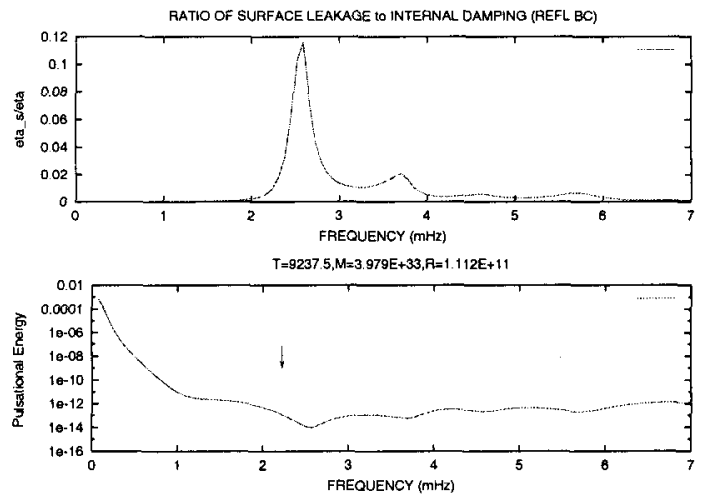

Figure 2. The top panel shows the ratio of surface to total losses. The bottom panel shows the normalised pulsation energy. 


\section{Other results}

We find that displacement nodes start appearing in the photosphere at frequencies larger than $2 \mathrm{mHz}$. They can be found within a depth of $4000 \mathrm{~km}$ from the surface at frequencies larger than $1 \mathrm{mHz}$. The temperature nodes start appearing within $4000 \mathrm{~km}$ from the surface at frequencies larger than $1 \mathrm{mHz}$, but there is a frequency range $(1.4 \mathrm{mHz}<\nu<1.8 \mathrm{mHz})$ where the first temperature node appears much deeper. The condition for temperature and displacement nodes to appear in the photosphere is $n>20$, where $n$ is the overtone.

The phase difference between temperature perturbations and displacement, $\Psi_{T}$, shows variations within the photosphere. It varies by as little as $9^{\circ}$ at $\nu=1 \mathrm{mHz}$ to as large as $285^{\circ}$ at $\nu=3 \mathrm{mHz}$.

\section{Conclusions}

Our calculations indicate the presence of a chromospheric mode in roAp stars [we note that evidence for chromospheres in the A stars $\alpha$ Aql, $\alpha$ Cep and $\tau^{3}$ Eri was reported by Simon \& Landsman (1997)]. The change in the phase difference between relative temperature and displacement eigenfunctions from the surface down to the photosphere can be very large; this must be taken into account in modelling the observed pulsation phase relations.

We caution that our results are based on models that do not include convection and magnetism. In particular, Balmforth et al. (2001) demonstrated that the interplay between magnetism and convection in roAp stars can lead to excitation of some modes through driving in the polar regions. However, we expect that the atmospheric behaviour found in the present calculations will nonetheless be representative of more realistic models.

\section{References}

Balmforth, N.J., Cunha, M.S., Dolez, N., Gough, D.O., \& Vauclair, S. 2001, MNRAS, 323, 362

Christensen-Dalsgaard, J. \& Frandsen, S. 1983, Solar Phys., 82, 165

Medupe R., Christensen-Dalsgaard, J., \& Kurtz, D.W. 1998, in ASP Conf. Ser., Vol. 135, A Half Century of Stellar Pulsation Interpretations: a Tribute to Arthur Cox, eds. P.A. Bradley \& J. Guzik, (San Francisco: ASP), 197

Simon, T. \& Landsman, W.B. 1997, ApJ, 483, 435

Watson, R.D. 1988, Ap\&SS, 140, 255 